\title{
Percepção dos Pacientes sobre o Tratamento em Serviços de Saúde Mental: Validação da Escala de Mudança Percebida
}

\author{
Patient's Perception on the Treatment in Mental Health Services: Validating \\ the Perception of Change Scale-Patient Version
}

\author{
Marina de Bittencourt Bandeira* ${ }^{*}$, , Mário César Rezende Andrade ${ }^{a}$, \\ Cecília Silva $\operatorname{Costa}^{b}, \&$ Mônia Aparecida da Silva ${ }^{a}$ \\ ${ }^{a}$ Universidade Federal de São João del-Rei, São João del-Rei, Brasil \\ $\&{ }^{b}$ Centro de Atenção Psicossocial, São João del-Rei, Brasil
}

\begin{abstract}
Resumo
Na avaliação de serviços de saúde mental, tem-se destacado as escalas de medida que avaliam os resultados do tratamento relatados pelos próprios pacientes. O objetivo desta pesquisa foi validar a Escala de Mudança Percebida (EMP) pelos pacientes. Trezentos pacientes psiquiátricos, atendidos em serviços de saúde mental, participaram de entrevistas individuais estruturadas, com a escala. A escala possui 19 itens que avaliam mudanças percebidas, com alternativas de resposta em escala do tipo Likert de 3 pontos. Análise pelo método dos Componentes Principais resultou em estrutura de 3 fatores: a) Ocupação e saúde física; b) Aspectos psicológicos e sono; c) Relacionamentos e estabilidade emocional. A escala apresentou boa consistência interna (Alfa de Cronbach $=0,85)$, estabilidade temporal teste-reteste $(r=0,93 ; p<0,05)$ e validade convergente com uma escala que avalia o construto teoricamente relacionado de satisfação com o serviço $(r=0,37 ; p<0,05)$.

Palavras-chave: Resultados relatados pelos pacientes; Percepção de mudança; Serviços de Saúde Mental; Escala de Medida.

Abstract

The evaluation of mental health services has stressed the use of patient-reported outcome scales. Thus, this research aimed at validating the Perception of Change Scale - Patient Version (PCS-P). Three hundred psychiatric patients, attending mental health services, participated in individual structured interviews which use the scale. The scale has 19 items which evaluate perceived changes and response alternatives disposed in a three-point Likert-type scale. Analysis using the Principal Components Method revealed a 3-factor structure: (a) occupation and physical health; (b) psychological aspects and sleeping condition; (c) relationships and emotional stability. The scale had good indices of internal consistency (Cronbach alpha $=$ $0.85)$, test-retest temporal stability $(r=0.93 ; p<0.05)$ and convergent validity with another scale evaluating the related construct of service satisfaction $(r=0.37 ; p<0.05)$.

Keywords: Patient-reported outcome; Perception of change; Mental Health Services; Scale.
\end{abstract}

O desenvolvimento de pesquisas avaliativas e de monitoramento dos serviços de saúde mental tem sido recomendado, visando promover a qualidade destes serviços (World Health Organization [WHO], 2001). As dimensões estrutura-processo-resultados têm sido consideradas como os pilares da avaliação de serviços de saúde em geral (Donabedian, 1966/2005). Dentre estas três dimensões, a avaliação dos resultados do tratamento tem sido priorizada pelos pesquisadores, tradicionalmente através de medidas objetivas aferidas por terceiros.

\footnotetext{
"Endereço para correspondência: Universidade Federal de São João Del Rei, Laboratório de Pesquisa em Saúde Mental, Praça Dom Helvécio, 74, Campus Dom Bosco, São João Del Rei, MG, Brasil, CEP 36300-000. E-mail: marina@mgconecta.com.br
}

Nas últimas três décadas, incluir a perspectiva dos próprios pacientes, na avaliação dos serviços de saúde mental, constituiu uma abordagem que tem despertado, cada vez mais, o interesse dos clínicos, dos administradores e das agências de credenciamento de serviços (Holcomb, Parker, Leong, Thiele, \& Higdon, 1998). Segundo Donabedian (1992), os resultados da assistência em saúde devem estar congruentes com a perspectiva dos usuários, senão a qualidade do tratamento ficaria comprometida. A visão de que os pacientes devem ser considerados como participantes ativos, em seu tratamento, estimulou a utilização de medidas subjetivas (McCabe, Saidi, \& Priebe, 2007). Foram desenvolvidas, em consequência, medidas de auto-relatos dos próprios pacientes sobre sua percepção a respeito dos resultados do tratamento nos serviços, também conhecidas como instru- 
mentos PRO ([patients reported outcomes], Fakhoury, Kaiser, Roeder-Wanner, \& Priebe, 2002; McCabe et al., 2007; United States Food and Drugs Administration [FDA], 2006).

A avaliação dos serviços de saúde mental tem sido proposta sob a forma de uma abordagem integrativa, que inclui a participação dos três agentes envolvidos nos serviços: usuários, familiares e profissionais do serviço (WHO, 2001). Dentre as recomendações apresentadas por Hansson (2001), para a avaliação de serviços de saúde mental, destaca-se igualmente a utilização de múltiplas perspectivas de medida e o uso concomitante de escalas objetivas e subjetivas, pois as medidas subjetivas estão mais relacionadas com a utilização dos serviços.

Este tipo de avaliação fornece informações que não estão presentes nas medidas clínicas dos profissionais, tais como mudanças nos eventos privados (ex. sintomas, sentimentos, disposição), no funcionamento do paciente e no seu bem-estar, tal como percebido por ele (McCabe et al., 2007). Tait e Lester (2005) destacam que o paciente tem um conhecimento único da vivência da doença mental, dos seus sintomas, das suas necessidades e da experiência com os serviços de saúde mental e uma perspectiva diferente da visão dos profissionais e que esta informação pode ser usada para aprimorar o tratamento. Segundo Hunter e Cameron (2008), as medidas da percepção dos pacientes, sobre os efeitos do tratamento, devem ser tomadas rotineiramente nos serviços de saúde mental e o estudo destes autores, ao longo de 3 anos, junto a pacientes com esquizofrenia, demonstrou ser isto possível.

Segundo Hasler, Moergeli e Schnyder (2004), as medidas subjetivas dos resultados são importantes, porque muitas vezes não há uma correspondência direta entre o que os usuários percebem como mudança desejável e os resultados objetivos mensurados por terceiros. Segundo Mercier, Landry, Cobiere e Perreault (2004), as medidas subjetivas avaliam o impacto real de mudanças produzidas pelo tratamento na vida dos pacientes, podendo calibrar as medidas objetivas de resultados do tratamento, aferidas por terceiros, como um indicador da validade social das intervenções efetuadas.

Incluir a percepção dos pacientes, na avaliação do tratamento, pode contribuir para aumentar sua auto-estima e o sentimento de controle ou emponderamento (empowerment), ao perceber que seu ponto de vista está sendo considerado (Tait \& Lester, 2005). Pode ainda aumentar a sua satisfação com os serviços, o que tem sido associado com uma melhor adesão ao tratamento e maior utilização dos serviços, diminuindo a taxa de abandono (Hansson, 2001; Hasler, Moergeli, Bachmann, et al., 2004; Ruggeri, 1994), além de ser um fator preditivo para menor taxa de hospitalizações futuras (Priebe \& Gruyters, 1995). A adesão ao tratamento é um fator essencial para a obtenção de resultados positivos das intervenções, especialmente em saúde mental, onde a falta de insight e os sintomas positivos e negativos dos pacientes resultam em uma dificuldade em aceitar ou dar continuidade à medi- cação e, portanto, medidas que estimulam a adesão devem ser priorizadas (Hunter \& Cameron, 2008).

A percepção dos pacientes de que ocorreram mudanças positivas em suas vidas, em função do tratamento recebido nos serviços, é importante, porque ela interfere na relação entre resultados objetivos do tratamento e o grau de satisfação dos usuários. Segundo Hasler, Moergeli, Bachmann, et al. (2004), se ocorrem mudanças objetivas, aferidas pelos profissionais, mas que não são percebidas pelos usuários como mudanças efetivas positivas em suas vidas, elas não terão efeito na sua satisfação com os serviços, o que explicaria as baixas correlações encontradas entre estas duas medidas.

A presente pesquisa teve como objetivo fazer a validação da Escala de Mudança Percebida (EMP), originalmente desenvolvida por Mercier et al. (2004), no Canadá e adaptada para o Brasil por Bandeira, Calzavara, Costa e Cesari (2009), a qual avalia os resultados do tratamento em serviços de saúde mental, em termos das mudanças percebidas pelos próprios pacientes, em diversas dimensões de sua vida. Este estudo faz parte de um projeto de pesquisa mais amplo, visando validar duas escalas de mudança percebida, nas versões dos pacientes e dos familiares, assim como investigar os fatores associados à percepção de mudança (Bandeira, 2007).

\section{Método}

\section{Participantes}

Participaram desta pesquisa 300 pacientes atendidos nas seguintes instituições psiquiátricas: Centros de Atenção Psicossocial das cidades de São João Del Rei, Lavras e Barbacena; Núcleo de Saúde Mental de São João Del Rei e Ambulatório de Psiquiatria do Departamento Municipal de Saúde Pública de Barbacena. A distribuição dos pacientes por cidade foi a seguinte: $40 \%$ na cidade de São João Del Rei, 38\% em Lavras e 22\% em Barbacena. Foram incluídos, na amostra, pacientes psiquiátricos que estavam em tratamento há, pelo menos, um ano no serviço. Foram excluídos aqueles pacientes que estavam em crise, ou possuíam co-morbidade de dependência química ou retardo mental associado ao problema psiquiátrico e aqueles que apresentaram dificuldade de responder às questões da escala. $\mathrm{O}$ tamanho da amostra utilizada se situa amplamente acima do mínimo recomendado na literatura da área, que requer, pelo menos, 5 a 10 vezes o numero de itens da escala (Artes, 1998; Dancey \& Reidy, 2006; Pasquali, 1999) e, no mínimo, 200 sujeitos (Pasquali, 1999).

A maioria dos participantes da pesquisa era do sexo feminino (66\%), com idade média de 42,12 anos, na faixa entre 18 e 74 anos. A média de escolaridade era de 6 anos. Grande parte dos pacientes tinha renda individual mensal de 1 a 2 salários mínimos (80\%). Os diagnósticos estavam distribuídos nas seguintes categorias: Esquizofrenia, Transtornos Esquizotípicos e Transtornos Delirantes (45\%), Transtornos Afetivos ou do Humor (40\%) e Trans- 
tornos Neuróticos, Relacionados ao Stress e Somatoformes (15\%), classificados segundo a Classificação Internacional de Doenças (CID-10) da Organização Mundial de Saúde (OMS, 1998). Os pacientes estavam doentes há 12,71 anos, em média. Quase a metade dos pacientes $(47,7 \%)$ já havia sido internada, com uma média de 4,17 internações. Metade dos pacientes (50\%) teve crises no último ano, com uma freqüência de 3,86 crises, em média. A maior parte dos entrevistados (91\%) estava em regime de atendimento não intensivo, com consulta médica mensal e o restante $(9 \%)$ estava utilizando o serviço do CAPS-dia, com frequência de uma vez por semana.

Uma sub-amostra de 40 pacientes participou de um estudo da estabilidade temporal da escala. A maioria era do sexo masculino (70\%), com idade média de 41,98 anos. Os pacientes tinham uma média de 5,08 anos de escolaridade e renda média de $\mathrm{R} \$ 257,95$ por mês. A duração média da doença era de 15,32 anos. Mais da metade dos pacientes (54\%) já havia sido internada, com uma média de 4,3 internações. Mais da metade dos pacientes (54\%) teve crises no último ano, sendo que o número médio de crises foi de 3,60. Com relação aos diagnósticos, $64 \%$ foram diagnosticados com Esquizofrenia, Transtornos Esquizotípicos e Transtornos Delirantes e o restante (36\%), com Transtornos do Humor e Afetivos, segundo a classificação do CID-10 (OMS, 1998).

Para avaliação da validade convergente, uma sub-amostra de 50 pacientes participou da aplicação de uma segunda escala de medida, avaliando a satisfação com os serviços de saúde mental (SATIS-BR). A maioria era de pessoas do sexo feminino (66\%), com idade média de 42,64 anos, renda individual mensal de 1 a 2 salários mínimos (67\%) e média de escolaridade de 6,17 anos. Os diagnósticos desta sub-amostra foram: Esquizofrenia, Transtornos Esquizotípicos e Transtornos Delirantes (34\%), Transtornos Afetivos ou do Humor (44\%) e Transtornos Neuróticos, Relacionados ao Stress e Somatoformes (22\%), segundo a classificação do CID-10 (OMS, 1998). A duração média da doença, nesta sub-amostra, era de 13,30 anos. Mais da metade já havia sido internada (54\%), com 4,32 internações, em média. Mais da metade dos pacientes (54\%) teve crises no último ano, com uma freqüência média de 3,60 crises. A maioria estava em tratamento não intensivo ( $88 \%$ ), indo ao serviço uma vez por mês, para consulta médica e avaliação. O restante (12\%) estava em tratamento semi-intensivo, que incluía, além da consulta médica, a participação no CAPS-dia, pelo menos uma vez por semana.

\section{Instrumentos de Medida}

Escala de Mudança Percebida (EMP). A versão original desta escala, "Questionaire of Perceived Changes", foi elaborada por Mercier et al. (2004), no Canadá, visando avaliar a percepção de mudanças pelos pacientes como resultado do tratamento em serviços de saúde mental. A escala original possui 20 itens, sendo que um item é global e os outros 19 itens avaliam a percepção dos pacientes sobre as mudanças ocorridas em três domínios de sua vida, segundo os fatores identificados pela análise fatorial: Self e Relacionamentos, Saúde Física e Condições de Vida. Quanto à consistência interna, o valor do coeficiente alfa de Cronbach para a escala global foi 0,92 e, para os três fatores, foram respectivamente $0,91,0,68$ e 0,82 . A escala original foi recentemente renomeada como Perceived Improvement Questionnaire (PIQ), por Perreault et al. (2010).

O instrumento foi adaptado para o Brasil, com as modificações necessárias, tendo-se demonstrado sua validade de conteúdo e sua equivalência semântica com o instrumento original, assim como a sua facilidade de compreensão pela população-alvo (Bandeira et al., 2009). A versão brasileira da escala EMP contém 19 itens, que avaliam a percepção do paciente sobre as mudanças ocorridas em sua vida como resultado do tratamento recebido, com alternativas de resposta dispostas em uma escala do tipo Likert de 3 pontos, sendo 1. Pior do que antes, 2. Sem mudança, 3. Melhor do que antes. A escala adaptada possui um item a menos, em relação à escala original, em função da análise feita pela comissão de especialistas, no processo de adaptação transcultural da escala, que o considerou inapropriado (ver detalhes em Bandeira et al., 2009). A aplicação da escala é feita através de uma entrevista, na qual as questões são lidas e as respostas anotadas pelo entrevistador. Dentre os 19 itens da escala, um item global avalia como o paciente percebe, em geral, os efeitos do tratamento recebido, enquanto os demais itens avaliam as mudanças percebidas em diversas dimensões de sua vida, incluindo aspectos físicos, psicológicos, vida social, condições de vida, etc. Esta escala está disponível no site do Laboratório de Pesquisa em Saúde Mental (LAPSAM) da Universidade Federal de São João Del Rei (UFSJ).

Escala de Satisfação dos Usuários de Serviços de Saúde Mental (SATIS-BR versão abreviada). Este instrumento foi elaborado originalmente pela OMS, como parte do projeto WHO-SATIS (WHO, 1996), que visava construir instrumentos de avaliação dos serviços de saúde mental, que incluíssem a avaliação da satisfação dos três agentes envolvidos nos serviços, pacientes, familiares e profissionais, assim como a sobrecarga sentida pelos profissionais e familiares. Sua adaptação e validação para o Brasil foram feitas em um estudo realizado por Bandeira, Pitta e Mercier (2000). A escala é composta por 13 questões, com alternativas de resposta dispostas em uma escala Likert de 5 pontos, na qual o valor 1 indica o menor grau de satisfação e o valor 5 indica o maior grau de satisfação com o serviço.

A escala possui dois fatores. O primeiro contém cinco questões que avaliam a satisfação do paciente com relação à competência da equipe, à maneira como foi acolhido, ao impacto do seu tratamento, às informações dadas pela equipe e ao grau em que a equipe discutiu ou não com ele sobre o seu tratamento. O segundo fator é composto por quatro questões que avaliam a satisfação do 
usuário com o conforto e a aparência do serviço, com as condições gerais das instalações do serviço e com as condições de sua admissão. Foram encontrados valores adequados de alfa de Cronbach para a escala global e para as sub-escalas. $O$ valor de alfa da escala global foi de $0,84 \mathrm{e}$ as correlações item-total variaram entre 0,26 e 0,69 . Para o primeiro fator, obteve-se um alfa igual a 0,77 , e correlações item-total entre 0,40 e 0,67 . O valor do alfa do segundo fator foi de 0,55 , e as correlações item-total variaram entre 0,22 e 0,49 . Na escala global, há ainda quatro questões adicionais, que se referem aos resultados do tratamento, à competência do terapeuta principal, à maneira de ser tratado no serviço e ao grau de compreensão da equipe sobre o problema do paciente (Bandeira et al., 2000).

Questionário Sócio-demográfico e Clínico. Foi utilizado um questionário visando avaliar as características sócio-demográficas e clínicas dos pacientes. Este questionário havia sido previamente testado em um estudo piloto, visando avaliar a adequação das questões para um bom entendimento pela população-alvo. As variáveis sócio-demográficas incluídas no questionário foram: idade, sexo, escolaridade e renda. As variáveis clínicas foram: diagnóstico, idade em que ficou doente, há quantos anos está doente, tempo de tratamento, existência de outra doença além do transtorno psiquiátrico, dados sobre internações em hospital psiquiátrico, tipo e freqüência da medicação, dados sobre a ocorrência de crises no ano anterior, uso de bebidas ou de drogas.

\section{Procedimentos}

Coleta de Dados. Após aprovação do projeto pelos serviços de saúde mental e pela Comissão de ética da UFSJ, os participantes foram contatados para participarem da pesquisa. As entrevistas foram realizadas na própria instituição ou na casa do entrevistado, conforme sua disponibilidade. Antes do início de cada entrevista, eram explicados ao paciente os objetivos da pesquisa, o procedimento, o anonimato dos dados e sua liberdade de interromper a qualquer momento a realização da entrevista, se desejasse desistir da sua participação. Era explicado, ainda, que não havia respostas certas ou erradas e que ele deveria apenas responder, de forma sincera, de acordo com a sua opinião. Durante as entrevistas, os entrevistadores aplicavam a escala EMP e o questionário sócio demográfico, lendo as questões para os participantes e marcando suas respostas.

Duas sub-amostras de pacientes foram selecionadas aleatoriamente da amostra total. Para estudo da validade convergente, uma sub-amostra de 50 pacientes participou, na mesma entrevista, da aplicação da escala SATIS-BR, realizada logo após a aplicação da escala EMP. Para o estudo da estabilidade temporal da escala EMP, uma segunda sub-amostra, de 40 sujeitos, participou de uma reaplicação desta mesma escala, em uma entrevista que foi realizada após um intervalo de 3 semanas (Vallerand, 1989).
Análise de Dados. Os procedimentos adotados para verificação da validade de construto da escala EMP foram a análise fatorial e a análise por hipóteses. Para a análise da estrutura fatorial da escala, foram explorados dois métodos, o de Componentes Principais e o método de Análise fatorial pelos eixos principais (Dancey \& Reidy, 2006), utilizando-se as rotações Varimax e a Oblimin, com e sem restrição de fatores. Foi utilizado o critério mínimo de 0,40 de carga fatorial para retenção dos itens na escala (Dancey \& Reidy, 2006). O critério para delimitação do número de fatores a serem retidos foi definido com base no valor do eigenvalue superior a 1,0, na análise gráfica do Scree Test e na presença de, pelo menos, três itens com cargas acima do mínimo estabelecido (Zwick \& Velicer, 1986). Além destes critérios, foi levada em consideração a estrutura fatorial da escala original, que incluía três fatores, a fim de verificar qual solução seria teoricamente mais interpretável.

O segundo procedimento realizado foi a Análise por Hipóteses, enfocando a técnica de validação convergente. Este procedimento visa verificar a correlação da escala com uma outra que avalia um construto distinto, porém, teoricamente relacionado (Pasquali, 2003). Foi utilizado o construto de satisfação com os serviços, pois, no estudo realizado por Holcomb et al. (1998), estes autores constataram que a melhora percebida pelos pacientes entre o pré e o pós-teste era um importante preditor do sentimento posterior de satisfação com os serviços. Tendo em vista esta relação observada entre percepção de mudança e satisfação com o serviço, foi escolhida uma escala de satisfação para avaliar a validade convergente da escala EMP. Foi selecionada a Escala de Satisfação dos Usuários de Serviços de Saúde Mental (SATIS-BR), já validada para o Brasil. A análise dos dados foi feita através do cálculo da correlação de Pearson entre os escores destas duas escalas de medida.

A fidedignidade da escala EMP foi avaliada por meio de dois procedimentos: a análise de sua consistência interna e a análise de sua estabilidade temporal. A avaliação da estabilidade temporal da escala foi feita por meio da análise de Correlação Intraclasse (ICC), entre os escores obtidos no teste e no reteste, de uma mesma sub-amostra. A consistência interna da escala global e dos fatores foi avaliada através do coeficiente alfa de Cronbach. Foi utilizado como critério mínimo para retenção dos itens na escala, o valor de 0,20 de correlação item-total (Likert, 1932). Para a realização destas análises estatísticas foi utilizado o programa estatístico SPSS-PC, versão 7.5.

\section{Resultados}

A análise da estrutura fatorial mostrou que a solução teoricamente consistente e interpretável resultou da utilização do método de Componentes Principais e rotação Varimax, com restrição de três fatores (Tabela 1). As demais soluções apresentaram indicadores teoricamente ou estatisticamente inferiores, tais como cargas fatoriais 
muito baixas, fatores com menos de três itens, composição dos itens menos adequada teoricamente. Os indicadores obtidos de adequação da amostra foram: $\mathrm{KMO}=$ 0,88 ; Bartlet $=1216,90 ; p<0,01$. A estrutura fatorial final, com três fatores, com valores de eigenvalues acima de 1,0 , explicou $42,13 \%$ da variância dos dados, tendo todos os itens cargas fatoriais acima de 0,47 .

O primeiro fator da escala contém oito itens, que avaliam as mudanças percebidas pelos pacientes com relação às suas ocupações e sua saúde física, explicando 18,80\% da variância dos dados. O segundo fator contém seis itens, que avaliam as mudanças percebidas pelos pacientes na dimensão psicológica e na qualidade do sono, explicando $14,10 \%$ da variância dos dados. O terceiro fator da escala contém quatro itens, que avaliam as mudanças percebidas pelos pacientes nos seus relacionamentos e na sua estabilidade emocional, explicando $9,23 \%$ da variância dos dados.

Tabela 1

Análise Fatorial da Escala de Mudança Percebida (EMP) pelos Pacientes

\begin{tabular}{|c|c|c|c|}
\hline Itens / Fatores & $\begin{array}{l}\text { Atividades e } \\
\text { Saúde Física }\end{array}$ & $\begin{array}{c}\text { Aspectos } \\
\text { Psicológicos } \\
\text { e Sono }\end{array}$ & $\begin{array}{c}\text { Relacionamentos } \\
\text { e Estabilidade } \\
\text { Emocional }\end{array}$ \\
\hline 16. Atividades de lazer & 0,66 & & \\
\hline 8. Energia & 0,65 & & \\
\hline 17. Tarefas de casa & 0,63 & & \\
\hline 18. Capacidade de cumprir as obrigações e tomar decisões & 0,61 & & \\
\hline 15. Interesse em trabalhar ou se ocupar com alguma coisa & 0,55 & & \\
\hline 11. Sexualidade & 0,54 & & \\
\hline 7. Apetite & 0,48 & & \\
\hline 10. Saúde Física & 0,46 & & \\
\hline 4. Sentimento de confiança em si próprio & & 0,65 & \\
\hline 2. Humor & & 0,60 & \\
\hline 1. Problemas pessoais & & 0,60 & \\
\hline 5. Sentimento de interesse pela vida & & 0,53 & \\
\hline 6. Capacidade de suportar situações difíceis & & 0,52 & \\
\hline 9. Sono & & 0,48 & \\
\hline 13. Convivência com amigos e amigas & & & 0,63 \\
\hline 3. Estabilidade das emoções & & & 0,60 \\
\hline 14. Convivência com outras pessoas & & & 0,48 \\
\hline 12. Convivência com a família & & & 0,47 \\
\hline
\end{tabular}

Notas. $\mathrm{KMO}$ medida de adequação da amostra $(\mathrm{KMO}=0,88)$; Teste de Bartlet: qui quadrado $=1216,90 ; p<0,01$; Variância explicada $=42,13 \% ; * p<0,01$.

A Tabela 2 apresenta as correlações de Spearman dos fatores entre si e com a escala global. Foi utilizada esta análise estatística, tendo em vista que a amostra não apresentou distribuição normal, pelo teste KolmogorovSmirnov (K-S=1,98; $p<0,001)$. Pode-se notar que todas as correlações foram significativas e positivas e que as correlações entre cada fator e a escala global foram mais elevadas do que aquelas entre os fatores. Assim, embora os fatores representem dimensões distintas, todos estão relacionados a um construto comum, presente na Escala de Mudança Percebida (EMP).

Tabela 2

Correlações entre os Fatores da Escala EMP dos Pacientes

\begin{tabular}{lccc}
\hline \multicolumn{1}{c}{ Fatores } & $\begin{array}{c}\text { Fator1 } \\
\text { Atividades e saúde fisica }\end{array}$ & $\begin{array}{c}\text { Fator 2 } \\
\text { Aspectos psicológicos } \\
\text { e sono }\end{array}$ & $\begin{array}{c}\text { Fator 3 } \\
\text { Relacionamentos e } \\
\text { estabilidade emocional }\end{array}$ \\
\hline Fator 1 & 1 & $0,53^{*}$ & $0,50^{*}$ \\
Fator 2 & $0,53^{*}$ & 1 & $0,43^{*}$ \\
Fator 3 & $0,50^{*}$ & $0,43^{*}$ & 1 \\
Escala Global & $0,92^{*}$ & $0,74^{*}$ & $0,71^{*}$ \\
\hline
\end{tabular}

Nota. ${ }^{*} p<0,01$. 
Bandeira, M. B., Andrade, M. C. R., Costa, C. S. \& Silva, M. A. (2011). Percepção dos Pacientes sobre o Tratamento em Serviços de Saúde Mental: Validação da Escala de Mudança Percebida.

No que se refere à validade convergente da escala EMP, os resultados mostraram uma correlação positiva significativa $(r=0,37 ; p<0,05)$ entre os escores obtidos com a aplicação da escala EMP e os dados obtidos com uma medida de um construto distinto, porém teoricamente relacionado, que se refere à satisfação dos pacientes com os serviços onde receberam o seu tratamento, avaliada por meio da escala SATIS-BR.

A fidedignidade da escala foi avaliada através da análise de sua consistência interna e da sua estabilidade tem- poral. Os resultados referentes à consistência interna da escala EMP global e dos fatores separadamente, analisada pela análise de alfa de Cronbach, estão apresentados na Tabela 3. Os dados mostram, para a escala global, um valor de alfa de 0,84 e correlações item-total que se situa-ram entre 0,20 e 0,60 . Para o fator 1 , obteve-se um valor de alfa de 0,78 , com correlações item-total entre 0,34 e 0,60 . Para o fator 2 , obteve-se um valor de alfa de 0,67 , com correlações item- total entre 0,28 a 0,53 . O terceiro fator apresentou um valor de alfa de 0,55 , com correlações item-total de 0,20 e 0,41 .

Tabela 3

Coeficientes Alfa de Cronbach por Fator, Correlações Item-total, Valores de Alfa Para cada Item da Escala de Mudança Percebida pelos Pacientes

\begin{tabular}{|c|c|c|c|}
\hline Fator (Valor de Alfa) & Item & Correlação item-total & Valor de Alfa sem o item \\
\hline $\begin{array}{l}\text { Atividades } \\
\text { e Saúde Física } \\
(\alpha=0,78)\end{array}$ & $\begin{array}{l}\text { Atividades de lazer } \\
\text { Energia } \\
\text { Tarefas de casa } \\
\text { Capacidade de cumprir } \\
\text { obrigações e tomar decisões } \\
\text { Interesse em trabalhar ou se } \\
\text { ocupar com alguma atividade } \\
\text { Sexualidade } \\
\text { Apetite } \\
\text { Saúde Física }\end{array}$ & $\begin{array}{l}0,50 \\
0,35 \\
0,40 \\
0,34\end{array}$ & $\begin{array}{l}0,75 \\
0,77 \\
0,77 \\
0,78\end{array}$ \\
\hline $\begin{array}{l}\text { Aspectos } \\
\text { Psicológicos } \\
\text { e Sono } \\
(\alpha=0,67)\end{array}$ & $\begin{array}{l}\text { Confiança em si mesmo } \\
\text { Humor } \\
\text { Problemas pessoais } \\
\text { Interesse pela vida } \\
\text { Capacidade de suportar situações difíceis } \\
\text { Sono }\end{array}$ & $\begin{array}{l}0,53 \\
0,46 \\
0,37 \\
0,43 \\
0,36 \\
0,28\end{array}$ & $\begin{array}{l}0,58 \\
0,61 \\
0,64 \\
0,62 \\
0,65 \\
0,67\end{array}$ \\
\hline $\begin{array}{l}\text { Relacionamentos } \\
\text { e Estabilidade } \\
\text { Emocional } \\
(\alpha=0,55)\end{array}$ & $\begin{array}{l}\text { Convivência com amigos(as) } \\
\text { Estabilidade das emoções } \\
\text { Convivência com outras pessoas } \\
\text { Convivência com familiares }\end{array}$ & $\begin{array}{l}0,41 \\
0,20 \\
0,39 \\
0,35\end{array}$ & $\begin{array}{l}0,41 \\
0,59 \\
0,53 \\
0,46\end{array}$ \\
\hline \multicolumn{2}{|c|}{ Escala Global $(\alpha=0,84)$} & - & - \\
\hline
\end{tabular}

Nota. ${ }^{*} p<0,01$.

Tabela 4

Médias e Desvios-padrão dos Escores das Sub-escalas e da Escala Global no Teste e no Reteste, Após 3 semanas e Coeficientes de Correlação intra-Classe (ICC) entre os Dois Escores

\begin{tabular}{|c|c|c|c|}
\hline Sub-escalas e Escala Global & $\begin{array}{l}\text { Aplicações } \\
\text { da EMP }\end{array}$ & $\begin{array}{c}\text { Médias e } \\
\text { Desvios padrão (DP) }\end{array}$ & $\begin{array}{c}\text { Correlações } \\
\text { Teste-Reteste (ICC) }\end{array}$ \\
\hline \multirow[t]{2}{*}{ Ocupação e saúde física } & Teste & $2,34(0,43)$ & \multirow[t]{2}{*}{$0,85^{*}$} \\
\hline & Reteste & $2,38(0,39)$ & \\
\hline \multirow[t]{2}{*}{ Dimensão psicológica e sono } & Teste & $2,78(0,24)$ & \multirow[t]{2}{*}{$0,80 *$} \\
\hline & Reteste & $2,71(0,29)$ & \\
\hline \multirow[t]{3}{*}{ Relacionamentos e estabilidade emocional } & Teste & $2,43(0,39)$ & \multirow[t]{3}{*}{$0,79 *$} \\
\hline & Reteste & $2,47(0,36)$ & \\
\hline & Teste & $2,55(0,28)$ & \\
\hline Escala global & Reteste & $2,54(0,28)$ & $0,93^{*}$ \\
\hline
\end{tabular}

Nota. ${ }^{*} p<0,01$ 
A Tabela 4 apresenta os resultados referentes à análise da estabilidade temporal da escala, através da correlação intraclasse, entre os escores do teste e do reteste. Os dados mostram uma correlação positiva significativa entre os escores das duas aplicações da escala, tanto para a escala global $(r=0,93 ; p<0,01)$, quanto para os três fatores, respectivamente $(r=0,85 ; r=0,80 ; r=0,79 ; p<0,05)$. A escala EMP se encontra no Anexo deste artigo.

\section{Discussão}

Os resultados obtidos indicam que a escala EMP apresenta qualidades psicométricas adequadas, com relação à sua validade de construto, validade convergente, sua consistência interna e estabilidade temporal. $\mathrm{Na}$ análise fatorial da escala, obteve-se uma matriz com a extração de três fatores, que apresentaram a solução mais adequada estatística e teoricamente. Os valores obtidos do coeficiente alfa de Cronbach indicam que os itens de cada fator e da escala global são homogêneos, possuindo consistência interna, com valores acima de 0,70 para a escala global e o primeiro fator, situando-se assim dentro da faixa considerada ideal por Vallerand (1989), entre 0,70 e 0,85 . Os fatores 2 e 3 apresentaram valores de alfa abaixo deste valor, porém ainda acima de 0,50 , o que é considerado aceitável (Bowling, 1997). As correlações itemtotal dos itens de cada fator foram todas acima de 0,20 , critério mínimo adotado para retenção dos itens na escala (Perreault, Katerelos, Sabourin, Leichner, \& Desmarais, 2001). Os resultados referentes ao procedimento de correlação teste-reteste indicam uma boa estabilidade temporal da escala EMP, uma vez que todos os escores obtidos (na faixa de 0,79 a 0,93 ) tanto para a escala global quanto para os fatores, se situaram acima de 0,60 , valor este considerado mínimo para esta propriedade psicométrica (Vallerand, 1989).

$\mathrm{Na}$ validação da escala original canadense (Mercier et al., 2004), também foram encontrados três fatores através da análise fatorial, porém com uma distribuição diferente dos itens, talvez devido a diferenças culturais ou às características clínicas diferenciadas da populaçãoalvo, que consistia de pacientes com diagnóstico de dependência química. Os valores de alfa de Cronbach, encontrados na presente pesquisa, foram menos elevados do que os obtidos no estudo da escala original, onde apenas um dos fatores teve valor de alfa abaixo de 0,70. Esta diferença é comumente encontrada em pesquisas de adaptação transcultural de instrumentos de medida (Vallerand, 1989).

Quanto à validade convergente da escala, foi obtida uma correlação positiva significativa com a SATIS-BR, confirmando a hipótese postulada de uma relação teórica entre os construtos de mudança percebida e de satisfação com o serviço. Este resultado comprova a validade convergente da escala EMP, uma vez que a escala foi capaz de comprovar a hipótese testada. A relação das mudanças percebidas pelos pacientes com sua satisfação em relação ao tratamento recebido nos serviços tem sido evidenciada por estudos desenvolvidos nos últimos anos, tais como as pesquisas de Hasler, Moergeli, Bachmann, et al. (2004).

\section{Conclusões}

Pode-se concluir que a escala EMP possui propriedades psicométricas adequadas para sua aplicação no contexto brasileiro, podendo ser utilizada para avaliar a percepção de mudanças pelos próprios pacientes, em função do tratamento recebido nos serviços de saúde mental. Sendo uma escala multifatorial, ela se torna mais sensível para avaliar mudanças percebidas em dimensões distintas da vida dos pacientes, o que possibilita obter resultados diferenciados, em função de cada dimensão avaliada, contrariamente às escalas unifatoriais que apresentam menor sensibilidade (Ruggeri, 1994). A escala permite ainda identificar em que aspectos estão ocorrendo melhoras percebidas ou, ao contrário, estão ocorrendo pioras ou ausência de mudança, possibilitando assim utilizar esta informação para redirecionar e aprimorar o tratamento visando resultados mais adequados. Os dados obtidos com a escala EMP poderão ser úteis para complementar as avaliações dos profisssionais e ajudar o serviço a oferecer tratamentos mais adequados às necessidades dos pacientes. Tendo em vista a sua forma curta e a facilidade de aplicação e de compreensão da escala pela população-alvo, ela poderia ser aplicada nos serviços de saúde mental, de forma rotineira, tal como sugerido por Hunter e Cameron (2008), autores que já demonstraram ser isto possível em seu estudo longitudinal.

\section{Referências}

Artes, R. (1998). Aspectos estatísticos da análise fatorial de escalas de avaliação. Revista de Psiquiatria Clínica, 25(5), 223-228.

Bandeira, M. (2007). Integrando a percepção dos usuários na avaliação dos serviços de saúde mental. Manuscrito nãopublicado, Universidade Federal de São João Del Rei, MG.

Bandeira, M., Calzavara, M. G. P., Costa, C. S., \& Cesari, L. (2009). Avaliação de serviços de saúde mental: Adaptação transcultural de uma medida da percepção dos usuários sobre os resultados do tratamento. Jornal Brasileiro de Psiquiatria, 58(2), 107-114.

Bandeira, M., Pitta, A. M. F., \& Mercier, C. (2000). Escala de Avaliação da Satisfação dos Usuários em Serviços de Saúde Mental. Jornal Brasileiro de Psiquiatria, 49(8), 293-300.

Bowling, A. (1997). Measuring disease: A review of disease specific quality of life measurement scales. Buckingham, UK: Open University Press.

Dancey, C. P., \& Reidy, J. (2006). Introdução à análise de fatores. In C. P. Dancey \& J. Reidy, Estatística sem Matemática para Psicologia (3. ed., pp. 420-455). Porto Alegre, RS: Artmed.

Donabedian, A. (1992). Quality assurance in heath care: Consumer's role. Quality in Health Care, 1, 247-251. 
Donabedian, A. (2005). Evaluating the quality of medical care. The Milbank Quartely, 83(4), 691-729. (Original work published 1966)

Fakhoury,W. K. H., Kaiser, W., Roder-Wanner, U. U., \& Priebe, S. (2002). Subjective evaluation: Is there more than one criterion? Schizophrenia Bulletin, 28(2), 319-327.

Hansson, L. (2001). Outcome assessment in psychiatric service evaluation. Social Psychiatry and Psychiatric Epidemiology, 36(5), 244-248.

Hasler, G., Moergeli, H., \& Schnyder, U. (2004). Outcomes of psychiatric treatment: What is relevant for our patients? Comprehensive Psychiatry, 45(3), 199-205.

Hasler, G., Moergeli, H., Bachmann, R., Lambreva, E., Buddeberg, C., \& Schnyder, U. (2004). Patient satisfactions with outpatient psychiatric treatment: The role of diagnosis, pharmacotherapy, and perceived therapeutic change. The Canadian Journal of Psychiatry, 49(5), 315-321.

Holcomb, W. R., Parker, J. C., Leong, G. B., Thiele, J., \& Higdon, J. (1998). Customer satisfaction and self-reported treatment outcomes among psychiatric inpatients. Psychiatric Services, 49(7), 929-934.

Hunter, R., \& Cameron, R. (2008). The Scottish Schizophrenia Outcomes Study: A new paradigm for utilizing self-report assessments from patients with schizophrenia. European Psychiatric Review, 1(2), 7-9.

Likert, R. (1932). A technique for the measurement of attitudes. Archives of Psychology, 140, 55.

McCabe, R., Saidi, M., \& Priebe, S. (2007). Patient-reported outcomes in schizophrenia. British Journal of Psychiatry, 50(Suppl.), S21-S28.

Mercier, L., Landry, M., Corbiere, M., \& Perreault, M. (2004). Measuring client's perception as outcome measurement. In A. R. Roberts \& K. R. Yeager. Evidence-based practice manual: Research and outcome measures in health and human services. Oxford, UK: Oxford University Press.

Organização Mundial de Saúde. (1998). CID-10: Classificação Internacional de Doenças e Problemas Relacionados à Saúde (10. ed. rev.). São Paulo, SP: Editora da Universidade de São Paulo.

Pasquali, L. (1999). Testes referentes a construto: Teoria e modelo de construção. In L. Pasquali (Ed.), Instrumentos psicológicos: Manual prático de elaboração (cap. 3, pp. 5675). Brasília, DF: Laboratório de Pesquisa em Avaliação e Medida.

Pasquali, L. (2003). Validade dos testes. In L. Pasquali, Psicometria: Teoria dos testes na Psicologia e na Educação (cap. 6, pp. 158-188). Petrópolis, RJ: Vozes.

Perreault, M., Katerelos, T. E., Sabourin, S., Leichner, P., \& Desmarais, J. (2001). Information as a distinct dimension for satisfaction assessment of outpatient psychiatric services. International Journal of Health Care Quality Assurance, 14(2), 111-120.

Perreault, M., White, N. D., Fabre, E., Landry, M., Anestin, A. S., \& Rabouin, D. (2010). Relationship between perceived improvement and treatment satisfaction among clients of a methadone maintenance program. Evaluation and Program Planning, 33(4), 410-417.

Priebe, S., \& Gruyters, T. (1995). Patients'assessment of treatment predicting outcome. Schizophrenia Bulletin, 21(1), 87-94.

Ruggeri, M. (1994). Patients' and relatives' satisfaction with psychiatric services: The state of the art of its measurement. Social Psychiatry and Psychiatric Epidemiology, 29(5), 212-227.
Tait, L., \& Lester, H. (2005). Encouraging user involvement in mental health services. Advances in Psychiatric Treatment, $11,168-175$.

United States Food and Drugs Administration. (2006). Guidance for Industry. Patient-Reported Outcome Measures: Use in Medical Product Development to Support Labeling Claims. Health Qual Life Outcomes, 4, 79.

Vallerand, R. J. (1989). Vers une méthodologie de validation trans-culturelle de questionnaires psychologiques: implications pour la recherché en langue française. Canadian Psychology, 30(4), 662-680.

World Health Organization. (1996). WHO-SATIS. Consumer's and caregiver's satisfaction with mental health services: A multisite study. Geneva, Switzerland: Author.

World Health Organization. (2001). The World Health Report. Mental health: New understanding, new hope. Geneva, Switzerland: Author.

Zwick, W. R., \& Velicer, W. F. (1986). Comparaison of five rules for determining the number of components to retain. Psychological Bulletin, 99(3), 432-442.
Recebido: 28/09/2009

$1^{a}$ revisão: $29 / 01 / 2010$ Aceite final: 12/03/2010 
Anexo

\section{ESCALA DE MUDANÇA PERCEBIDA (EMP) \\ (VERSÃO DO PACIENTE)}

1. Você acha que o tratamento que você está recebendo aqui o ajudou a se sentir melhor?

Se Sim: Como?

Se Não: Por quê?

2. Agora, eu vou lhe perguntar, para cada aspecto da sua vida, se você acha que você teve mudanças desde que começou a se tratar aqui no (nome do local) e se estas mudanças foram para pior ou para melhor.

Nota ao entrevistador: Para cada item abaixo, dizer : "Desde que você começou a se tratar aqui, ........ está(ão).....”.

Seus problemas pessoais

2. Seu humor

3. A estabilidade das suas emoções

4. Sua confiança em você mesmo

5. Seu interesse pela vida

6. Sua capacidade de suportar situações difíceis

7. Seu apetite

8. Sua energia (disposição para fazer as coisas)

9. Seu sono

10. Sua saúde física (Dores, tremores, etc.)

11. Sua sexualidade (satisfação sexual)

12. Sua convivência com sua família

13. Sua convivência com seus amigos ou amigas

14. Sua convivência com as outras pessoas

15. Seu interesse em trabalhar ou se ocupar com alguma coisa

16. Suas atividades de lazer (as coisas que você gostava de fazer)

17. Suas tarefas de casa (ex: cozinhar, fazer compras para a casa, lavar roupa, arrumar o quarto ou a casa, consertar coisas, etc).

$\begin{array}{rcr}\text { Pior do } & \text { Sem } & \text { Melhor do } \\ \text { que antes } & \text { mudança } & \text { que antes }\end{array}$

que antes

